\title{
Selection Methodologies of Materials and Manufacturing Processes
}

\author{
Sydney Ferreira Santos*, Maurizio Ferrante \\ Departamento de Engenharia de Materiais, Universidade Federal de São Carlos \\ 13565-905 São Carlos - SP, Brazil
}

Received: January 1, 2003; Revised: September 8, 2003

\begin{abstract}
The present work discusses some principles of materials selection and examines a few selection criteria and contexts in which this technical activity takes place. Emphasis is given to the concept of Merit Indices, and to the methodology employed for its deduction. The Materials Properties Charts and their integration with the Merit Indices are introduced and illustrated by the former of the two case studies here presented whilst the second associates the methodology of materials selection to process selection.
\end{abstract}

Keywords: materials selection, process selection, materials properties

\section{Introduction}

Materials selection (MS) is a multidisciplinary activity, which cuts across a large number of professional fields. As a consequence, it draws together people with different backgrounds ranging from the scientific to the quasi-technical, such as marketing for instance.

Motivations for MS can be either the realization of a completely new product or, more frequently, the substitution of an existing material. In the latter case, performance's improvement and cost reduction can be the main driving forces for selection, but malfunction, weight reduction, feasibility of recycling and processability, are also frequent motivations. For instance, weight reduction is one of the main targets for design improvements and MS, particularly in the automotive and aerospace industries ${ }^{1}$.

Generally any MS event must consider a large number of material candidates and premature exclusions must be avoided. Also, when facing a MS problem, engineers are normally asked to choose a solution which satisfies more than one objective, that is, not only lower weight, but (for instance) also low cost, good fatigue resistance, better fabricability etc. To overcome these difficulties the material engineer has to observe a number of principles and rules ${ }^{2,3,4}$, some of which are summarized below

-Adoption of the so called compromise philosophy: it is well known that the ideal material for a given application does not exist, and the best is the one which displays the better combination of useful properties. Indeed, there are properties inversely correlated, such as yield strength and fracture toughness.

- At the early stages of any selection process a macroscopic approach must be adopted, mainly in order of not to miss any opportunity. Along the way, new restrictions and additional criteria will be applied to the initial group of candidates, restricting it further and further until the final choice can be made. Past experience, embodied in computer-based systems called $e x$ pert systems, can be employed to narrow the initial range of candidate materials ${ }^{5}$.

- The path from macroscopic to microscopic or detailed approach can be shortened if a reasoned choice of the restrictions is adopted. For instance, starting with a minimum fracture toughness level or a maximum operating temperature allows early elimination of a very large number of materials. Generalizing: it is better to initiate the selection process with non-negotiable properties (that is, requiring the yes or no type of answer) than with properties which exhibit a range of values, can be modified by heat treatment, surface modification, etc. Typically, the non-negotiable material properties are qualitative: oxidation resistance; corrosion resistance; radiation sensitivity, workability, machinability. To evaluate and compare properties classified as qualitative (for MS purposes), adjectives (excellent, good, inadequate, etc.) or grades (10, 8,7 , etc.) can be attributed.

- Interaction of material selection with process selection (PS) is always a critical point in any product de- 
sign and realization. The difficulties increase when material and process are interrelated variables. For instance, consider the case in which it was found that material $\mathrm{A}$ is more suitable than material $\mathrm{B}$ but the former cannot be processed as easily than the latter, if large quantities of the item are required. In this particular case it may be advisable to start by selecting the process, say, powder metallurgy. The range of candidate materials will thus be restricted to those which can be processed by that technology.

- Adoption of a formalization methodology by which materials are ranked according to some criteria. This is usually the last step of a MS procedure and its use is mandatory when a large number of materials is involved and/or conflicting materials demands are present. Today there are many techniques which can be employed for the purpose: (i) decision matrices ${ }^{6}$; (ii) decision making theory ${ }^{7}$ and control area diagrams ${ }^{8}$.

The two last mentioned principles - MS / PS interaction and formalization methodology, form the subject of the present paper and will be developed using two case studies.

\section{Merit Indices and Materials Properties Maps}

\subsection{Merit indices (MI)}

This is one of the most important concepts of MS. It is an algebraic formula expressing a compromise between two materials properties. In its simplest form a MI can be the property itself, (or the inverse of it), but in most cases it is written as a fraction, where the numerator is the quantity one wishes to maximize and the denominator the quantity to be minimized. In other world a MI is a coupled equation and its use allows a quick comparison of alternatives without having to resort to mechanical design calculations.

For instance, taking strength as the design criteria and low weight as the critical MS requirement, it can be shown that the resulting MI is $\left[\sigma^{n} / \rho\right]$, where $n$ is a function of loading mode, and the most suitable candidate material is the one in which that ratio is maximized. Alternatively, if two properties have to be simultaneously maximized, then the MI is expressed by their product. Therefore, for each case it is possible to identify or deduce one (or more) suitable MI. This methodology is quite simple in some cases but can be difficult in others, mainly when multiple objectives are involved.

\subsection{Materials Properties Charts (MPC)}

These are bi-dimensional graphic representations in which the axes are assimilated to materials properties plotted on logarithmic scales. The range of the axes is chosen to include all materials, but of course regions of interest can be enlarged at will. These maps were introduced by M.F. Ashby in a pioneering paper ${ }^{9}$ and constitute one of the most powerful tools for MS. Recently, the MPCs were integrated with a number of databases forming the CES4.1 software commercialized by GRANTA DESIGN ${ }^{10}$. Integration of the MPC with MI is a very useful MS tool, and a number of recent papers and textbooks employ the MPC+MI methodology and provide many application examples ${ }^{11,12,13}$.

\section{Case Studies}

Material selection - agricultural implement: this equipment is attached to the back of a tractor and is designed to distribute pesticide in liquid form (spray) on plantations. It consists of a tank and two long structural arms which support pipes provided with a number of spray nozzles. The attributes of the structural arms are low weight, capacity to distribute the product on a large area and reasonable stiffness. Additional supports are to be avoided because of complexity of assembly, simplicity of maintenance and easy storage when the equipment is not in use. The cost has to be competitive with the existing solution, which is a carbon steel. Summarizing:

Product: support of fertilizer sprayers, see Fig. 1;

Shape and sizes: pipe with outside diameter $\left(\mathrm{D}_{\mathrm{o}}\right)$, thickness ( $\mathrm{t}$ ) and length (L) equal to $0.10 \mathrm{~m} ; 3 \times 10^{-3} \mathrm{~m}$ and $5.00 \mathrm{~m}$, respectively;

Selection motivation: replacement of low carbon steel by a more suitable material.

Batch number: 50 pairs.

Objectives: low weight, controlled deflection, reasonable cost.

Candidate materials: aluminum alloy 6061T4, GFRP (70\% fibers type $\mathrm{S}$ in polyester matrix), CFRP (70\% continuous and unidirectional fibers in polyester matrix).

MI deduction: the system can be simplified to a cantilever beam (with cylindrical hollow shape) submitted to a uniform load. Bending stress and deflection of the beam are mainly due to its own weight.

Objective equation:- minimum mass (m)

$$
\mathrm{m}=\rho \cdot \mathrm{V} \approx \rho \cdot \mathrm{L} \cdot(2 \cdot \pi \cdot \mathrm{r} \cdot \mathrm{t})
$$

where $\mathrm{V}$ is volume, $\rho$ the material's density and $\mathrm{r}$ the arm average radius $\left(\approx \mathrm{r}_{\mathrm{o}}\right)$.

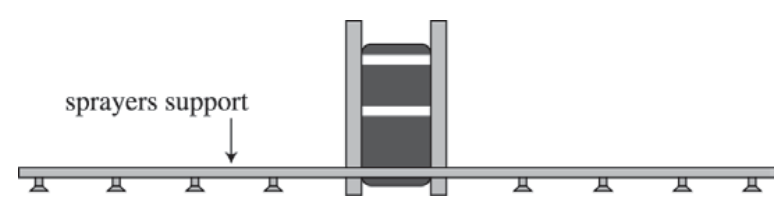

Figure 1. Schematic representation of the agricultural implement. 


\section{Restriction equation:}

$$
\mathrm{Y}_{\max }=\frac{\mathrm{W} \cdot \mathrm{L}^{3}}{8 \cdot \mathrm{E} \cdot \mathrm{I}}
$$

where $\mathrm{Y}_{\max }$ is the deflection of the arm, $\mathrm{W}$ the concentrated load (equal to $\mathrm{W} \mathrm{L}$, where $\mathrm{W}$ is the distributed weight per unit length), $\mathrm{L}$ the length, E the Young's modulus and I the moment of inertia. The value of I for a hollow circular cross section shape is given by:

$$
\mathrm{I}=\frac{\pi}{4}\left(\mathrm{r}_{\mathrm{o}}^{4}-\mathrm{r}_{\mathrm{i}}^{4}\right) \approx \pi \cdot \mathrm{r}^{3} \cdot \mathrm{t}
$$

Substituting Equation 3 in 2 we have:

$$
\mathrm{Y}_{\max }=\frac{\mathrm{W} \cdot \mathrm{L}^{3}}{8 \cdot \mathrm{E} \cdot\left(\pi \cdot \mathrm{r}^{3} \cdot \mathrm{t}\right)}
$$

The free variable is $r$, and eliminating it between the
Eqs. 1 and 4, results:

$$
\mathrm{m}=\left(\frac{\mathrm{W} \cdot \mathrm{L}^{7} \cdot \pi^{2} \cdot \mathrm{t}^{2}}{\mathrm{Y}_{\max }}\right)^{1 / 3} \cdot\left[\frac{\rho}{\mathrm{E}^{1 / 3}}\right]
$$

and the MI is equal to: $\left[\frac{E^{1 / 3}}{\rho}\right]$

Figure 2 is a MPC of the Young Modulus (E) vs. density ( $\rho)$ on which a straight line representing the MI above deduced has been superimposed. The axes of the chart are logarithmic, hence to each pair of $\mathrm{E}$ and $\rho$ the MI corresponds to a unique constant value $\mathrm{C}$ :

$$
\log \mathrm{Y}=\log \mathrm{X}+3 \log \mathrm{C}
$$

In the present case $Y=E$ and $X=\rho$; therefore this relationship plots as a straight line with slope 3 . This line was drawn passing through the LC (low carbon) steels position

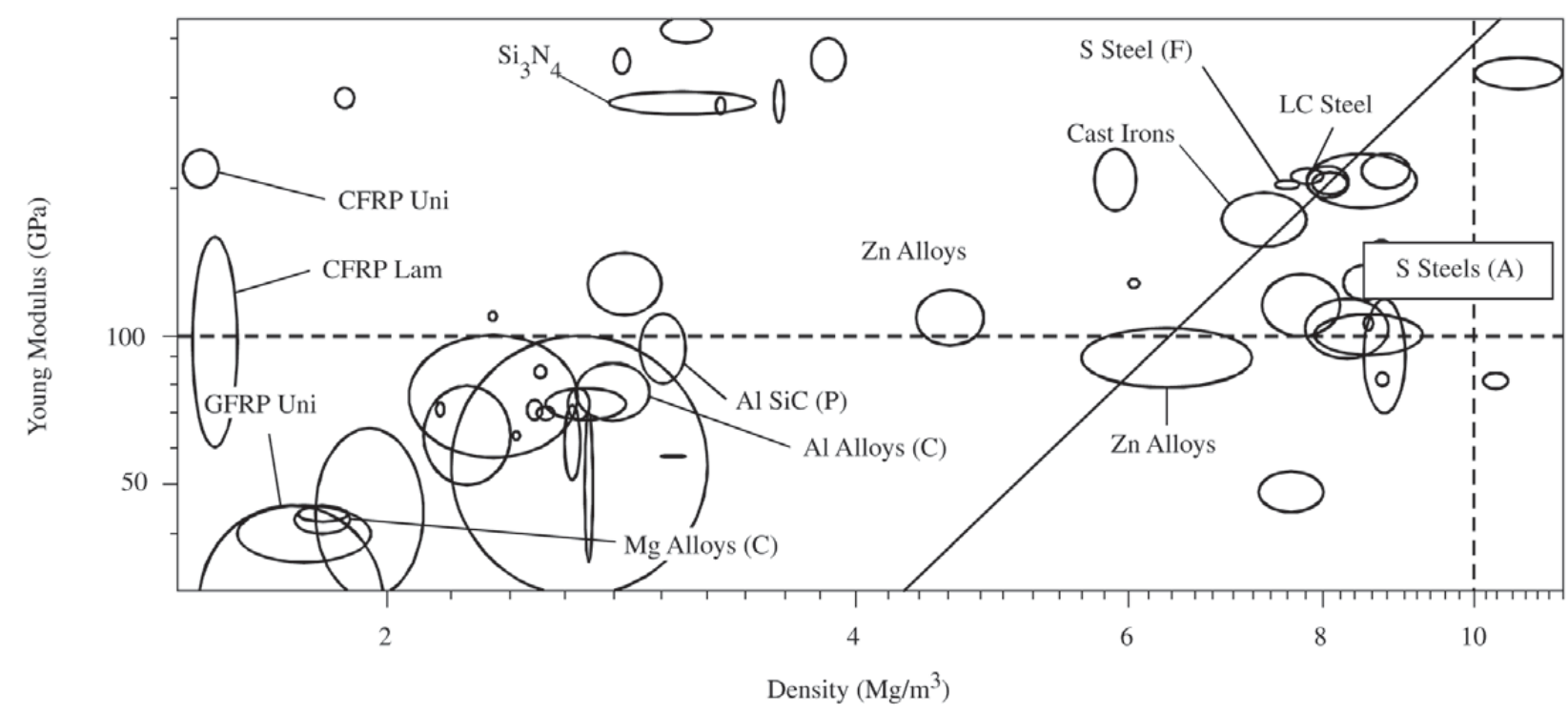

Figure 2. A region of the Young Modulus versus density map.

Table 1. Relevant properties of selected materials and performance data.

\begin{tabular}{ccccccc}
\hline $\begin{array}{c}\text { Materials } \\
\left(\mathrm{Mg} / \mathrm{m}^{3}\right)\end{array}$ & $\begin{array}{c}\rho \\
(\mathrm{GPa})\end{array}$ & $\begin{array}{c}\mathrm{E} \\
(\mathrm{US} \$ \mathrm{~kg})\end{array}$ & $\begin{array}{c}\text { Cost } \\
(\mathrm{kg})\end{array}$ & $\begin{array}{c}\text { Mass } \\
(\mathrm{US} \$ / \mathrm{unit})\end{array}$ & $\begin{array}{c}\text { Product Cost } \\
\text { deflection }(\mathrm{mm})\end{array}$ & Maximum \\
\hline $\mathrm{CFRP}$ & 1.5 & 70 & 16.50 & 7.10 & 117.00 & 13 \\
GFRP & 1.9 & 26 & 3.60 & 8.90 & 32.00 & 44 \\
Al alloy & 2.8 & 80 & 3.40 & 13.20 & 45.00 & 21 \\
Carbon Steel & 7.8 & 210 & 1.00 & 36.70 & 37.00 & 23 \\
\hline
\end{tabular}


in the map, since this is the reference material. Integration between MI and MPC means that the line can be shifted parallel to itself (thus maintaining slope 3) assuming different $\mathrm{C}$ values, each corresponding to the materials through which the line passes. The graph shows clearly that the lowest weight is obtained with CFRP since its MI is larger than those of the other candidates.

The relevant properties and the performance of the candidate materials are summarised in Table 1. It must be observed that in the present case 'performance' is represented by the weight and by the deflection of the pipes. The two last parameters were calculated employing Eqs. 1 and 4.

From the above data the formalization procedure of this case study can be carried out, and for this purpose the Pahl $\&$ Beitz decision matrix was chosen ${ }^{14}$. The man features of this method are summarized below:

(i) A list of criteria is generated and each criteria is weighted numerically such that the sum of all weighting factors equals 1 ;

(ii) If there are several criteria in the selection procedure, an "objective tree" can be constructed to help to assign the weighting factors. In this simple case study it will not be necessary.

(iii) A matrix of alternatives and criteria is constructed supposing there are $k$ materials candidates and $n$ criteria. The matrix is completed by assigning a value $\mathrm{v}$ to each criterion for each alternative. These values can be assigned as follows: 0 - unsatisfactory; 1 - just tolerable; 2 - adequate; 3 - good; and 4 - very good.

(iv) The number of values to assign is $(k) .(n)$. Each value is represented by $\mathrm{v}_{\mathrm{ij}}$. Once all values are assigned, the $(k) .(n)$ weighted values $(\mathrm{v} \mathrm{w})_{\mathrm{ij}}$ are computed by multiplying each value $\mathrm{v}_{\mathrm{ij}}$ by the associated criteria weighting factor $w_{\mathrm{ij}}$. Summing all weighted values for alternative $i$ produces the overall weighted value $\left(\mathrm{OWV}_{\mathrm{i}}\right)$. It may be useful to point out that if a criteria depends sequentially from another and each is characterized by a weigth factor, $\mathrm{w}_{\mathrm{A}}$ and $\mathrm{w}_{\mathrm{B}}$, the overall weight factor for criteria $A$ is of course $w_{A}$, but for criteria $B$ is $\mathrm{w}_{\mathrm{A}} \times \mathrm{w}_{\mathrm{B}}$. In these situations it is advisable to draw an 'objective tree' in order to identify clearly the relative dependence of criteria.

(v) $\mathrm{OWV}_{\mathrm{i}}$ is the raw score for a given alternative. It is customary to normalize the overall weighted values to 1 by dividing them by the product of the maximum value $\mathrm{v}_{\max }$ and the sum of the weighting factors. The formula of this weighted rating, $\mathrm{WR}_{\mathrm{i}}$, also known as the weighted property index, is:

$$
W R_{i}=\frac{\sum_{j=1}^{n}\left(w_{j} \cdot v_{i j}\right)}{v_{\max } \cdot \sum_{j=1}^{n} w_{j}}
$$

where normally $\sum_{j=1}^{n} w_{j}=1$.

Table 2 shows the results of the Pahl \& Beitz formalization procedure applied to the present case study. The largest OWR was achieved by GFRP, followed by the Al alloy, CFRP and carbon steel. These results must be considered carefully, by remembering that the weighting factors were arbitrarily assigned; indeed, the precise assignment of these factors is a critical procedure which will be briefly discussed later.

Process selection and material selection - brake cylinder

Product: brake cylinder - grey cast iron (SAE J431 SAE 3500),

Selection motivation: material substitution for better performance.

Batch number: 10.000 parts

Objectives: minimum weight, good surface finish, nearnet-shape characteristics, low cost

Candidate materials: aluminium alloy (A356), microalloyed steel (ASTM A487)

MI deduction: the system can be simplified to a cylinder with radius $\mathrm{R}$, lateral surface area $\mathrm{A}_{\mathrm{s}}$, wall thickness $\mathrm{t}$, supporting an internal pressure P. Therefore:

Table 2. Results of the Pahl \& Beitz method applied to the sprayer support.

\begin{tabular}{cccccccrrr}
\hline \multirow{2}{*}{ Criteria } & \multirow{2}{*}{$\mathrm{w}_{\mathrm{ij}}$} & \multicolumn{2}{c}{ Al alloy } & \multicolumn{2}{c}{ GFRP } & \multicolumn{2}{c}{ CFRP } & \multicolumn{2}{c}{ Steel } \\
& & $\mathrm{w}_{\mathrm{j}}$ & $\mathrm{w}_{\mathrm{i}} \mathrm{v}_{\mathrm{ij}}$ & $\mathrm{v}_{\mathrm{i}}$ & $\mathrm{w}_{\mathrm{i}} \mathrm{v}_{\mathrm{ij}}$ & $\mathrm{v}_{\mathrm{i}}$ & $\mathrm{w}_{\mathrm{i}} \mathrm{v}_{\mathrm{ij}}$ & $\mathrm{v}_{\mathrm{i}}$ & $\mathrm{w}_{\mathrm{i}} \mathrm{v}_{\mathrm{ij}}$ \\
\hline Low weight & 0.40 & 3.0 & 1.2 & 3.0 & 1.2 & 4.0 & 1.6 & 0.0 & 0.0 \\
Low cost & 0.40 & 2.0 & 0.8 & 4.0 & 1.6 & 0.0 & 0.0 & 4.0 & 1.6 \\
Low deflection & 0.20 & 4.0 & 0.8 & 3.0 & 0.6 & 4.0 & 0.8 & 4.0 & 0.8 \\
WR $_{\mathrm{j}}$ & & 2.8 & & 3.4 & & 2.4 & & 2.4 \\
\hline
\end{tabular}


Objective equation:

$$
m=A_{s} \cdot t \cdot \rho
$$

Restriction equation:

$$
\sigma_{1}=\frac{P \cdot R}{t}
$$

where $\sigma_{1}$ is the hoop stress. The free variable is t, and elimi-

Table 3. Relevant properties of the component constructed with the candidate materials

\begin{tabular}{cccc}
\hline & Cast iron & Alloy Steel & $\begin{array}{c}\text { Aluminium } \\
\text { Alloy }\end{array}$ \\
\hline$[\sigma / \rho]$ & 30 & 47 & 70 \\
Relative weight* & 1 & 0.65 & 0.40 \\
Relative part cost & 1 & 1.20 & 1.06 \\
\hline
\end{tabular}

*For equal strength nating it between the above equations, results:

$$
m=P \cdot A_{S} \cdot R \cdot\left[\frac{\rho}{\sigma}\right]
$$

and $\left[\frac{\sigma}{\rho}\right]$ is the MI.

Table 3 presents the MI, relative weight and cost of the component, constructed with each of the selected materials.

The aluminium alloy meets the objective if it can be assumed that the slightly higher cost with respect to cast iron is offset by the benefit of lower weight. Of course, in real situations this assumption must be supported by a comprehensive study which answers the following question: "how much is the customer willing to pay for a particular advantage (lower weight, for instance)".

Next step will deal with PS and the following options will be considered: Permanent mould casting; tixocasting and forging.

Table 4. Correlation of Processes, Product Specification and Properties

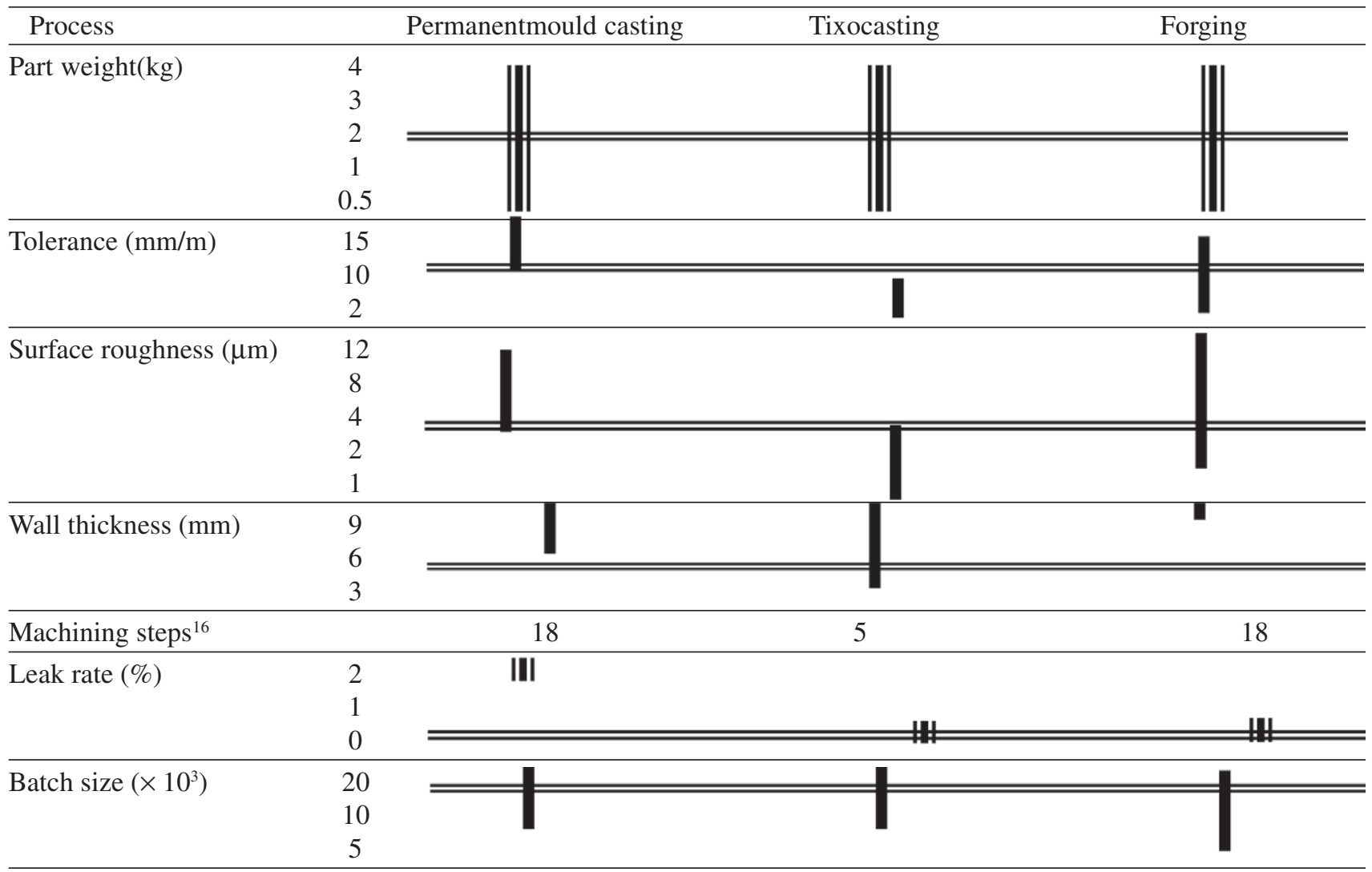


Product specifications are as follows:

Weight (max): $2.5 \mathrm{~kg}$; Tolerance: $10 \mathrm{~mm} / \mathrm{m}$; Surface finish: $3 \mu \mathrm{m}$; Wall thickness: $4.0 \mathrm{~mm}$.

Table 4 presents the correlation between processes (permanent mold casting, tixocasting and forging), product specifications and materials properties.

Analyzing the data it can be concluded that tixocasting is the more convenient process.

\section{Comments and Concluding Remarks}

The success of MS formalization procedures, be it the Pahl and Beitz model or any other technique, depends on the correct choice of the weighting factors, which must reflect the relative priority among the materials properties and/or MI. Of course, reliance on expert opinion can be very effective, but a number of novel approaches can improve the ranking procedure. For instance, the concept of entropy adapted by Shannon and Weaver to problems of information theory ${ }^{16}$ can be employed in the determination of weight factors ${ }^{7}$ Accordingly, the entropy $E_{j}$ of the normalized values of a $\mathrm{j}$-th material property $r_{i j}$ is given by:

$$
\mathrm{E}_{\mathrm{j}}=-\mathrm{k} \sum_{\mathrm{j}=1}^{\mathrm{m}} \mathrm{r}_{\mathrm{ij}} \log \mathrm{r}_{\mathrm{ij}}
$$

where $\mathrm{k}$ is $1 / \log \mathrm{m}$. It can be seen that when the property and/or the MI in consideration has a wide scatter, the entropy is low, that is, the information content is high; conversely, high entropy corresponds to a uniform set of data for the property/MI. The relationship between entropy and weight factor $\mathrm{w}_{\mathrm{j}}$ is defined by the expression:

$$
w_{j}=\frac{1-E_{j}}{\sum_{j=1}^{n}\left(1-E_{j}\right)}
$$

therefore, low entropy (large scatter) corresponds to large weight factors. This methodology can be improved by taking into account information coming from expert opinion; if to the latter a weight factor $\mathrm{w}^{*}$ can be attributed, the overall weight factor will be w, given by:

$$
\mathrm{W}^{*}=\frac{\mathrm{w}_{\mathrm{j}} \mathrm{w}_{\mathrm{j}} *}{\sum_{\mathrm{j}=1}^{\mathrm{n}} \mathrm{W}_{\mathrm{j}} \mathrm{w}_{\mathrm{j}} *}
$$

Summarizing, MS is a technical activity that bridges a number of engineering areas but it is also related to non-technical issues. In general terms, the methodology here presented is due to M.F. Ashby and although originally conceived as an educational tool, can be safely applied to real situations ${ }^{4}$. The MPC concept embodies the so called macroscopic approach and allows integration of the MI into the selection process.
The two case studies here presented were solved following the Ashby approach and are representative of typical MS events. In the former case it was shown how to deal with situations in which multiple selection criteria are present. The problem was solved by making use of a corresponding number of weighting factors and by applying the Pahl and Beitz decision matrix. The second case study dealt with a combination of MS and PS. This example could have been made more complex by introducing additional issues, such as the effect that different manufacturing processes can have on the material properties, fabricability differences among materials, such as machinability, weldability, and many others. Therefore, although it can be considered that presently MS counts with a relatively satisfactory methodology, there is still a lot of scope for a better integration between MS and PS.

\section{Acknowledgments}

The authors would like to thank Mr. F.J.X. de Carvalho (private consultant) for permission to publish case study № 1 .

\section{References}

1. Carle, D.; Blount, G. Materials and Design, v. 20, p. $267-$ 272, 1999.

2. Ferrante, M.; Santos, S.F.; de Castro, J.F.R. Materials Research n. 3, n. 2, p. 1-9, 2000.

3. Brechet, Y.; Bassetti, D.; Landru, D.; Salvo, L. Progress in Materials Science, v. 46, p. 407-428, 2001.

4. Ashby, M.; Brechet, Y.; Cebon, D. Advanced Engineering Materials, v. 4, n. 6, p. 327-334, 2002.

5. Er, R. Dias, Knowledge-Based Systems, v. 13, p. 225-234, 2000.

6. Bourrel, D.L. in ASM Handbook, Materials Selection and Design, v. 20, p. 291-296, 1997.

7. Jee, D-H.; Kang, K-J. Materials and Design, v. 21, p. 199-206, 2000.

8. R. Sandström, Materials and Design, v. 13, p. 195-202, 1992.

9. Ashby, M.F. Acta Metall.,v. 37, p. 1273-1293, 1989.

10. http://www.grantadesign.com

11. Ashby, M.F. Materials Selection in Mechanical Design, $2^{\text {nd }}$ Ed. Butterworth Heinemann, Oxford UK 1999.

12. Asbhy, M.F. Acta mater., v. 48, p. 359-369, 2000.

13. Charles, J.A.; Crane, F.A.A.; Furness, J.A.G. Selection and Use of engineering Materials, $3^{\text {rd }} \mathrm{Ed}$. Butterworth Heinemann, Oxford UK, 1997.

14. Pahl, G.; Beitz, W. Engineering Design: a Systematic Approach, Springer-Verlag, Germany, 1993.

15. Shannon, C.E.; Weaver, W. The Mathematical Theory of Communication. The University of Illinois Press, 1947.

16. Winterbottom, W.L. Metall. Sci. and Tech., v. 18, n. 2, p. 5-10, 2001 . 